\title{
LMI に基づく分散型アンチワインドアップ制御系設計
}

\author{
竹川正 浩* · 山 健*
}

LMI-based Decentralized Anti-windup Control Synthesis

Masahiro TAKEKAWA* and Tsuyoshi KIYAMA*

\begin{abstract}
This paper considers a decentralized anti-windup stabilizing control synthesis problem with a non-zero feedthrough matrix from output to input of saturation via the generalized sector approach. The sufficient solvability condition can be recast as a linear matrix inequality (LMI) one.
\end{abstract}

Key Words: saturation, dead-zone, domain of attraction, generalized sector, feedthrough matrix

\section{1.はじめに}

実際のシステムは，物理的な制約や制御対象の保護などの 理由から状態や入出力に拘束が存在する。このようなシステ ムに対して制御入力などの飽和を考慮せずに厳しい制御性能 を要求すると, ワインドアップ現象などの制御性能の劣化や 制御系の不安定化が引き起こされる場合がある. そこで, 線 形行列不等式 (LMI) に基づき, 集中型のアンチワインドアッ プ制御系設計が近年精力的に研究されてきている1).

本論文では飽和要素の出力から入力までの直達成分を考慮 する ${ }^{2)}$ アンチワインドアップ制御系の設計法の中で, 大規模 システムの制御に有効なように分散制御問題を考察する. 制 御対象の入出力チャンネル数と求めたいコントローラの次数 に応じてリヤプノフ行列に適切な構造をもたせることで $\left.{ }^{3)}, 4\right)$, 可解条件を LMI 条件の形式で導出できることを示す．また 数值例を用いてこの可解条件の妥当性を検証する.

表記 $: r$ 次元の実べクトルの集合を $\mathbb{R}^{r}, n \times m$ の実行列 の集合を $\mathbb{R}^{n \times m}, n \times m$ の零行列を $0_{n \times m}, n \times n$ の単位行 列を $I_{n}$ と記述する. 行列 $M$ に対し, $M^{\prime}$ は転置行列, $M^{i}$ は $i$ 行目の行ベクトル, $\operatorname{tr}(M)$ はトレースを表わす. 正方行 列 $M$ に対して, $\mathrm{He}(M):=M+M^{\prime}$ を定義する. 対称行列 $M$ に対して, $M>0(M \geq 0)$ は（準）正定行列を意味す る. 行列 $M_{1}, M_{2}$ に対する $\operatorname{diag}\left\{M_{1}, M_{2}\right\}$ をつぎのように 表記する.

$$
\operatorname{diag}\left\{M_{1}, M_{2}\right\}:=\left[\begin{array}{cc}
M_{1} & 0 \\
0 & M_{2}
\end{array}\right] .
$$

\section{2. 分散型アンチワインドアップ制御系}

Fig. 1 のフィードバック系で表わされる分散型アンチワイ ンドアップ制御系を考える。ただし， $G(s)$ は $N$ チャンネル の制御対象で,

\footnotetext{
* 大阪大学大学院工学研究科 吹田市山田丘 2-1

* Graduate School of Engineering, Osaka University, Suita (Received November 28, 2006)

(Revised June 6, 2007)
}

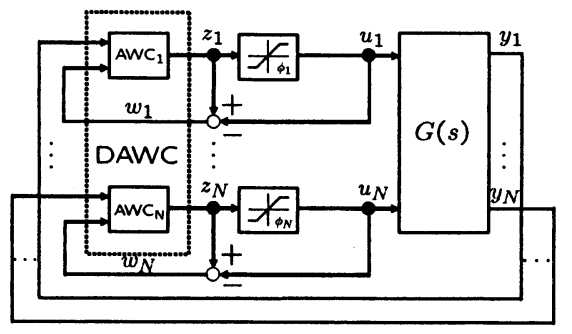

Fig. 1 Decentralized anti-windup control system

$$
\begin{aligned}
{\left[\begin{array}{c}
\dot{x} \\
y
\end{array}\right] } & =\left[\begin{array}{ll}
A & B \\
C & 0
\end{array}\right]\left[\begin{array}{l}
x \\
u
\end{array}\right], \\
B & :=\left[B_{1} B_{2} \cdots B_{N}\right], \quad C:=\left[C_{1}^{\prime} C_{2}^{\prime} \cdots C_{N}^{\prime}\right]^{\prime}, \\
u & :=\left[\begin{array}{lll}
u_{1}^{\prime} u_{2}^{\prime} \cdots u_{N}^{\prime}
\end{array}\right]^{\prime}, \quad y:=\left[y_{1}^{\prime} y_{2}^{\prime} \cdots y_{N}^{\prime}\right]^{\prime}
\end{aligned}
$$

により与えられ, 行列 $C_{i}$ は行フルランクとする. $x \in \mathbb{R}^{n}$, $u_{i} \in \mathbb{R}^{m_{i}}, y_{i} \in \mathbb{R}^{q_{i}}$ はそれぞれ状態変数, $i$ 番目のチャンネ ルの制御入力, $i$ 番目のチャンネルの観測出力, $m:=\sum_{i=1}^{N} m_{i}$ である. $\phi: \mathbb{R}^{m} \rightarrow \mathbb{R}^{m}$ は飽和関数であり,

$$
u=\phi(z) \Leftrightarrow u_{i}=\left\{\begin{aligned}
\sigma_{i} & \left(z_{i}>\sigma_{i}\right) \\
z_{i} & \left(\left|z_{i}\right| \leq \sigma_{i}\right) \\
-\sigma_{i} & \left(z_{i}<-\sigma_{i}\right)
\end{aligned}\right.
$$

のように定義される.ここで, $\sigma \in \mathbb{R}^{m}$ は各要素が正の与 えられたべクトルである．以下での記述を簡単にするため, Fig. 1 の制御系をループ変換しておく. つぎの不感帯関数 $\varphi: \mathbb{R}^{m} \rightarrow \mathbb{R}^{m}$,

$$
\varphi(z):=-\phi(z)+z
$$

を定義すると， $w=\varphi(z)$ とおくことにより，

$$
\begin{aligned}
{\left[\begin{array}{c}
\dot{x}_{c} \\
z
\end{array}\right] } & =\left[\begin{array}{cc:c}
A_{c} & B_{c 1} & B_{c 2} \\
C_{c} & D_{c 1} & D_{c 2}
\end{array}\right]\left[\begin{array}{c}
x_{c} \\
y \\
w
\end{array}\right]=: K\left[\begin{array}{c}
x_{c} \\
y \\
w
\end{array}\right], \\
A_{c} & :=\operatorname{diag}\left\{A_{c_{1}}, A_{c_{2}}, \cdots, A_{c_{N}}\right\} \\
B_{c 1} & :=\operatorname{diag}\left\{B_{c 1_{1}}, B_{c 1_{2}}, \cdots, B_{c 1_{N}}\right\} \\
B_{c 2} & :=\operatorname{diag}\left\{B_{c 2_{1}}, B_{c 2_{2}}, \cdots, B_{c 2_{N}}\right\} \\
C_{c} & :=\operatorname{diag}\left\{C_{c_{1}}, C_{c_{2}}, \cdots, C_{c_{N}}\right\} \\
D_{c 1} & :=\operatorname{diag}\left\{D_{c 1_{1}}, D_{c 1_{2}}, \cdots, D_{c 1_{N}}\right\}
\end{aligned}
$$




$$
\begin{aligned}
D_{c 2} & :=\operatorname{diag}\left\{D_{c 2_{1}}, D_{c 2_{2}}, \cdots, D_{c 2_{N}}\right\}, \\
x_{c} & :=\left[x_{c_{1}}^{\prime} x_{c_{2}}^{\prime} \cdots x_{c_{N}}^{\prime}\right]^{\prime}, \quad z:=\left[z_{1}^{\prime} z_{2}^{\prime} \cdots z_{N}^{\prime}\right]^{\prime}, \\
w & :=\left[w_{1}^{\prime} w_{2}^{\prime} \cdots w_{N}^{\prime}\right]^{\prime}
\end{aligned}
$$

の状態空間実現で与えられる分散型アンチワインドアップ コントローラ（DAWC）を考える.ただし， $x_{c_{i}} \in \mathbb{R}^{n_{c_{i}}}$, $w_{i} \in \mathbb{R}^{m_{i}}, z_{i} \in \mathbb{R}^{m_{i}}$ ，はそれぞれ $i$ 番目のチャンネルの DAWC の状態変数, 不感帯からの出力, DAWC からの出力 である。このとき，(1)，(3)，(4) 式より Fig.1 の閉ループ 系は

$$
\begin{aligned}
& {\left[\begin{array}{c}
\dot{\mathrm{x}} \\
z
\end{array}\right]=\left[\begin{array}{c:c}
\mathcal{A} & \mathcal{B} \\
\hdashline \mathcal{C} & \mathcal{D}
\end{array}\right]\left[\begin{array}{l}
\mathrm{x} \\
w
\end{array}\right]:=\left(L_{11}+L_{12} K L_{21}\right)\left[\begin{array}{l}
\mathrm{x} \\
w
\end{array}\right],} \\
& \mathrm{x}:=\left[\begin{array}{ll}
x^{\prime} & x_{c}^{\prime}
\end{array}\right]^{\prime}, \quad n_{c}:=\sum_{i=1}^{N} n_{c_{i}}, \quad q:=\sum_{i=1}^{N} q_{i}, \\
& \mathrm{~A}:=\left[\begin{array}{cc}
A & 0 \\
0 & 0_{n_{c} \times n_{c}}
\end{array}\right], \quad \mathrm{B}_{1}:=\left[\begin{array}{c}
-B \\
0_{n_{c} \times m}
\end{array}\right] \text {, } \\
& \mathrm{B}_{2}:=\left[\begin{array}{cc}
0 & B \\
I_{n_{c}} & 0
\end{array}\right], \quad \mathrm{C}_{1}:=\left[\begin{array}{cc}
0 & I_{n_{c}} \\
C & 0
\end{array}\right], \\
& C_{2}:=\left[\begin{array}{ll}
0 & I_{m}
\end{array}\right] \in \mathbb{R}^{m \times\left(n_{c}+m\right)} \text {, } \\
& {\left[\begin{array}{ll}
L_{11} & L_{12} \\
L_{21} & L_{22}
\end{array}\right]:=\left[\begin{array}{cc:c}
\mathrm{A} & \mathrm{B}_{1} & \mathrm{~B}_{2} \\
0 & 0 & \mathrm{C}_{2} \\
\hdashline \mathrm{C}_{1} & 0 & 0 \\
0 & I_{m} & 0
\end{array}\right]}
\end{aligned}
$$

と $\varphi$ のフィードバック結合で表現される. ここで, 行列 $\mathcal{D}$ は飽和要素の出力から入力までの直達成分に相当している.

\section{3. 吸収領域の解析}

与えられた実対称正定行列 $\mathcal{P}$ に対して,

$$
\mathcal{E}(\mathcal{P}):=\left\{x \in \mathbb{R}^{n}: x^{\prime} \mathcal{P}^{-1} x \leq 1\right\}
$$

の楕円領域を定義する。このとき，吸収領域はつぎのように 定義される。

【定義】実対対称正定行列 $\mathcal{P}$ が与えられているとする。こ のとき, $\mathcal{E}(\mathcal{P})$ 内の任意の点を初期值とする状態軌道がすべて の時間で $\mathcal{E}(\mathcal{P})$ 内に留まり，かつ時間の無限への経過に伴い 原点に収束する初期状態の領域 $\mathcal{E}(\mathcal{P})$ を吸収領域と定義する.

ここで, 与えられた行列 $N \in \mathbb{R}^{m \times\left(n+n_{c}\right)}$ と各要素が零以 上の值をもつ与えられたベクトル $\sigma \in \mathbb{R}^{m}$ に対して, 局所 領域

$$
\mathcal{L}(N, \sigma):=\left\{\mathrm{x} \in \mathbb{R}^{n+n_{c}}:\left\|N^{i} \mathrm{x}\right\| \leq \sigma_{i}, i=1, \ldots, m\right\}
$$

\section{を定義する。}

つぎに，本論文で用いるマルティプライア的手法 ${ }^{1)}$ を紹介 する.（7）式で定義される局所領域 $\mathcal{L}(N, \sigma)$ に対して，不感 帯関数（3）に関するつぎの命題 ${ }^{2}$ を導入する.

[命題 1] (3), (5) 式で定義される $H(s):=\mathcal{C}(s I-\mathcal{A})^{-1} \mathcal{B}+\mathcal{D}$ と $w=\varphi(z)$ のフィードバック結合を考える. 行列 $N \in$ $\mathbb{R}^{m \times\left(n+n_{c}\right)}$ が与えられているとする．このとき，

$$
\mathcal{D} T_{0}+T_{0} \mathcal{D}^{\prime}-2 T_{0}<0
$$

を満たす対角行列 $0<T_{0} \in \mathbb{R}^{m \times m}$ が存在するならば，任 意の対角行列 $0<T \in \mathbb{R}^{m \times m}$ に対して

$$
w^{\prime} T\{(N-\mathcal{C}) \times-(\mathcal{D}-I) w\} \leq 0 \quad{ }^{\forall} \times \in \mathcal{L}(N, \sigma)
$$

が成立する。

（注意）（8）式は, $H(s)$ と $\varphi$ の閉ループ系の Well-posedness 性の十分条件を与えるものであり, 詳細に関しては文献 2)を 参照されたい.

命題 1 および $S$-procedureにより証明される，楕円領域 (6) がシステム $H(s)$ と $\varphi$ の閉ループ系の吸収領域となる解 析の十分条件 ${ }^{1)}$ を紹介する.

[命題 2] $H(s):=\mathcal{C}(s I-\mathcal{A})^{-1} \mathcal{B}+\mathcal{D}$ と（3）式で定義され る $\varphi$ のフィードバック結合を考える：与えられた実対称行列 $\mathcal{P}>0$ に対して

$$
\begin{aligned}
\mathrm{He} & {\left[\begin{array}{cc}
\mathcal{A P} & \mathcal{B} S \\
\mathcal{C P}-\mathcal{R} & (\mathcal{D}-I) S
\end{array}\right]<0, } \\
& {\left[\begin{array}{cc}
\mathcal{P} & \mathcal{R}^{i \prime} \\
\mathcal{R}^{i} & \sigma_{i}^{2}
\end{array}\right] \geq 0 \quad{ }^{\forall} i=1, \ldots, m }
\end{aligned}
$$

を満たす対角行列 $S>0$ と行列 $\mathcal{R}$ が存在するならば, $\mathcal{E}(\mathcal{P})$ は吸収領域である。

\section{4. 吸収領域の設計}

Fig. 1 の閉ループ系に対して, コントローラの状態を零に 初期化したときの吸収領域は制限付吸収領域 ${ }^{1)}$ とよばれてい る.与えられた実対称正定行列 $X$ に対し，つぎの集合

$$
\mathcal{E}_{0}(X):=\left\{\left[\begin{array}{l}
x \\
0
\end{array}\right] \in \mathbb{R}^{n+n_{c}}: x^{\prime} X x \leq 1, x \in \mathbb{R}^{n}\right\}
$$

を定義する。このとき，吸収領域の部分集合である制限付吸 収領域 $\mathcal{E}_{0}(X)$ は吸収領域の定義と同様に定義される.

前章の解析の命題に基づいて, $\mathcal{E}(\mathcal{P}), \mathcal{E}_{0}(X)$ がそれぞれ吸 収領域, 制限付吸収領域となる DAWC の設計問題の可解条 件の十分条件は，つぎの LMI 条件で特徵づけられる.

《定理 1》 実対称行列 $X$, 各要素が正のベクトル $\sigma \in \mathbb{R}^{m}$ および制御対象（1）が与えられているとする。また，

$$
\begin{aligned}
\mathrm{C}_{1} U & :=\left[\begin{array}{ll}
I_{n_{c}+q} & 0
\end{array}\right], \mathbb{A}:=U^{-1} \mathrm{~A} U, \mathbb{B}_{1}:=U^{-1} \mathrm{~B}_{1}, \\
\mathbb{B}_{2} & :=U^{-1} \mathrm{~B}_{2}, U^{-1}=:\left[\begin{array}{ll}
T_{1} & T_{2}
\end{array}\right], \\
T_{1} & \in \mathbb{R}^{\left(n+n_{c}\right) \times n}, T_{2} \in \mathbb{R}^{\left(n+n_{c}\right) \times n_{c}}
\end{aligned}
$$

により行列 $U, \mathbb{A}_{1}, \mathbb{B}_{1}, \mathbb{B}_{2}, T_{1}, T_{2}$ を，(6)，(11) 式によ り集合 $\mathcal{E}(\mathcal{P}), \mathcal{E}_{0}(X)$ を定義する．このとき，

$$
\begin{aligned}
& \mathrm{He}\left[\begin{array}{cc}
\mathbb{A} P+\mathbb{B}_{2}\left[\begin{array}{ll}
V & 0
\end{array}\right] & \mathbb{B}_{1} S+T_{2} W \\
{\left[\begin{array}{lll}
V_{C} & V_{D} & 0
\end{array}\right]-R} & Q-S
\end{array}\right]<0, \\
& {\left[\begin{array}{cc}
P & R^{i \prime} \\
R^{i} & \sigma_{i}^{2}
\end{array}\right] \geq 0,\left[\begin{array}{cc}
P & T_{1} \\
T_{1}^{\prime} & X
\end{array}\right] \geq 0 \quad{ }^{\forall} i=1, \ldots, m}
\end{aligned}
$$




$$
\begin{aligned}
& P_{A}:=\operatorname{diag}\left\{P_{A 1}, \cdots, P_{A N}\right\}, \quad P_{A i} \in \mathbb{R}^{n_{c_{i}} \times n_{c_{i}}}, \\
& P_{B}:=\operatorname{diag}\left\{P_{B 1}, \cdots, P_{B N}\right\}, \quad P_{B i} \in \mathbb{R}^{n_{c_{i}} \times m_{i}}, \\
& P_{D}:=\operatorname{diag}\left\{P_{D 1}, \cdots, P_{D N}\right\}, \quad P_{D i} \in \mathbb{R}^{m_{i} \times m_{i}}, \\
& V_{A}:=\operatorname{diag}\left\{V_{A 1}, \cdots, V_{A N}\right\}, \quad V_{A i} \in \mathbb{R}^{n_{c_{i}} \times n_{c_{i}}}, \\
& V_{B}:=\operatorname{diag}\left\{V_{B 1}, \cdots, V_{B N}\right\}, \quad V_{B i} \in \mathbb{R}^{n_{c_{i}} \times q_{i}}, \\
& V_{C}:=\operatorname{diag}\left\{V_{C 1}, \cdots, V_{C N}\right\}, \quad V_{C i} \in \mathbb{R}^{m_{i} \times n_{c_{i}}}, \\
& V_{D}:=\operatorname{diag}\left\{V_{D 1}, \cdots, V_{D N}\right\}, \quad V_{D i} \in \mathbb{R}^{m_{i} \times q_{i}}, \\
& W:=\operatorname{diag}\left\{W_{1}, \cdots, W_{N}\right\}, \quad W_{i} \in \mathbb{R}^{n_{c_{i}} \times m_{i}}, \\
& Q:=\operatorname{diag}\left\{Q_{1}, \cdots, Q_{N}\right\}, \quad Q_{i} \in \mathbb{R}^{m_{i} \times m_{i}}, \\
& P_{1}:=\left[\begin{array}{cc}
P_{A} & P_{B} \\
P_{B}^{\prime} & P_{D}
\end{array}\right] \in \mathbb{R}^{\left(n_{c}+m\right) \times\left(n_{c}+m\right)}, \\
& P:=\left[\begin{array}{cc}
P_{1} & 0 \\
0 & P_{2}
\end{array}\right], P_{2} \in \mathbb{R}^{(n-m) \times(n-m)}, \\
& V:=\left[\begin{array}{cc}
V_{A} & V_{B} \\
V_{C} & V_{D}
\end{array}\right] \in \mathbb{R}^{\left(n_{c}+m\right) \times\left(n_{c}+q\right)}, \\
& \mathcal{P}:=U P U^{\prime}
\end{aligned}
$$

を満たす対角行列 $S>0$, 実対称行列 $P$, 行列 $Q, R, V, W$ が存在するならば, 吸収領域 $\mathcal{E}(\mathcal{P})$ と制限付吸収領域 $\mathcal{E}_{0}(X)$ を達成する分散型アンチワインドアップコントローラ (4) が 存在する。

（証明）命題の（9)，（10）式に $U^{-1}$ を用いる合同変換に より（12）式と（13）式の左の式を得る。また， $\mathcal{E}_{0}(X) \subseteq$ $\mathcal{E}_{0}\left(\left[\begin{array}{ll}I_{n} & 0\end{array}\right] \mathcal{P}^{-1}\left[\begin{array}{ll}I_{n} & 0\end{array}\right]^{\prime}\right)$ は (13) 式の右の式と等価である.

制約条件 (12)，（13）を満たす $X>0$ は一般に唯一でな く, 最大の制限付吸収領域 $\mathcal{E}_{0}(X)$ を与える $X$ を求めること が望ましい，このとき，楕円体 $\mathcal{E}_{0}(X)$ の大きさを測る尺度と して, たとえば $1 / \operatorname{tr}(X)$ を用いると, 本論文の枠組み内で最 大の制限付吸収領域を達成する DAWC の設計方法をつぎの ように得る。

\section{設計方法：}

1. LMI 条件を制約とする線形目的関数を最小化するつぎの 問題を解く.

$$
\underset{S>0, X, P, Q, R, V, W}{\operatorname{minimize}} \operatorname{tr}(X) \text { subject to (12), (13). (14) }
$$

2. DAWC の状態空間行列（4）をつぎのように計算する.

$$
\left[\begin{array}{ll:l}
A_{c} & B_{c 1} & B_{c 2} \\
C_{c} & D_{c 1} & D_{c 2}
\end{array}\right]=\left[\begin{array}{l:l}
V P_{1}^{-1} & W S^{-1} \\
& Q S^{-1}
\end{array}\right] .
$$

\section{5. 数 值 例}

定理 1 を用いることにより, Fig. 1 の閉ループ系を入力飽 和を許容しつつ安定化する DAWC が設計できる，以下では， 数值例を用いてこれを示す. 状態実現データ

$$
\left[\begin{array}{ll}
A & B \\
C & 0
\end{array}\right]=\left[\begin{array}{rrr:rrr}
0.006 & -0.016 & -0.053 & -0.696 & -1.326 \\
0.038 & 0.050 & -0.062 & 1.088 & -0.315 \\
0.007 & -0.030 & -0.002 & -0.547 & -0.921 \\
\hdashline-1.045 & 0.033 & 1.902 & 0 & 0 \\
-0.419 & 2.100 & 0.879 & 0 & 0
\end{array}\right]
$$
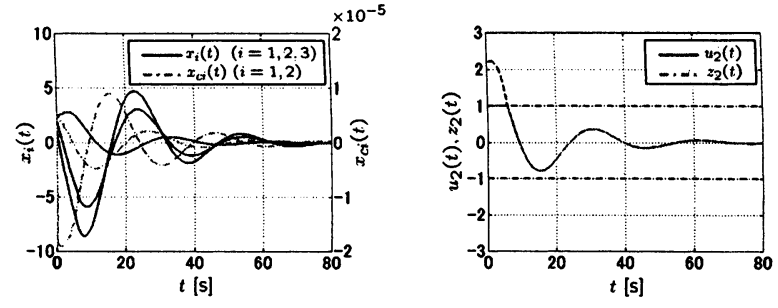

Fig. $2 x(t)$ and $x_{c}(t)$ vs. $t$

Fig. $3 u_{2}(t)$ and $z_{2}(t)$ vs. $t$

で与えられる不安定な制御対象（1）を考える．飽和リミット を $\sigma_{1}=1, \sigma_{2}=1$ とする. 状態の次数を $n_{c_{1}}=1, n_{c_{2}}=1$, 問題の簡単化のために行列 $C_{c}=I_{2}$ に規格化する可観測正準 形の DAWC を考える．このとき，リヤプノフ行列の一部を $P_{B}=0$ とし, 最小化問題 (14) を解くと,

$\left[\begin{array}{c:c}A_{c_{1}} & B_{c 1_{1}} B_{c 2_{1}} \\ \hdashline C_{c_{1}} & D_{c 1_{1}} D_{c 2_{1}}\end{array}\right]=\left[\begin{array}{c:cc}-8.23 \times 10^{5} & 1.45 & -3.28 \times 10^{-7} \\ \hdashline 1 & 0.19 & 1.00\end{array}\right]$,
$\left[\begin{array}{c:cc}A_{c_{2}} & B_{c 1_{2}} B_{c 2_{2}} \\ \hdashline C_{c_{2}} & D_{c 1_{2}} D_{c 2_{2}}\end{array}\right]=\left[\begin{array}{ccc}-8.36 \times 10^{5} & -2.60 & -3.35 \times 10^{-7} \\ \hdashline 1 & 0.28 & 0.43\end{array}\right]$, を得る。ここで, 閉ループ系の吸収領域内の初期状態

$$
\mathrm{x}=\left[\begin{array}{lll:ll}
0.96 & 2.29 & 2.01 & 0 & 0
\end{array}\right]^{\prime}
$$

の場合の状態と制御入力の時間応答をそれぞれ Fig. 2 と Fig. 3 に示す. Fig. 2 で, 実線は制御対象の状態 $x(t)$, 破 線は DAWC の状態 $x_{c}(t)$ である。また, Fig. 3 で, 実線は 制御入力 $u_{2}(t)$, 破線はコントローラ出力 $z_{2}(t)$ である.

明らかに, 制御入力が飽和を起こしても，吸収領域におい て閉ループ系を安定化させることが可能であることがわかる.

\section{6. おわりに}

入力飽和を有する分散型アンチワインドアップ制御系の安 定化問題について, 制御対象の入出力チャンネル数と求めた いコントローラの次数に応じてリヤプノフ行列に適切な構造 をもたせることで, 可解条件を LMI 条件の形式で導出でき ることを示した.

(注意) 定理 1 が成立するならば, 制御対象の固定モード は安定である，逆は成立しないことに注意されたい，

謝辞 有益な御意見を頂きました大阪大学大学院の池田雅 夫教授, 大阪府立大学大学院の Guisheng Zhai 准教授と査読 者の方々に感謝いたします。

\section{参 考 文 献}

1) K. Sawada and T. Kiyama: Relation between Single-stage and Two-stage Method for Anti-windup Control Synthesis, Proc. of SICE Annual Conf., 3027/3032 (2005)

2) T. Hu, A.R. Teel and L. Zaccarian: Performance analysis of saturated systems via two forms of differential inclusions, Proc. of CDC-ECC, 8100/8105 (2005)

3) M. Mattei: Sufficient Condition for the Synthesis of $\mathcal{H}_{\infty}$ Fixed-order Controllers, Internationl Journal of Robust and Nonlinear Control, 10, 1237/1248 (2000)

4) S. Murao, G. Zhai and M. Ikeda: Decentralized $\mathcal{H}_{\infty}$ controller design; an LMI approach, Proc. of SICE Annual Conf. 2002 in Osaka (2002) 